\title{
Multifractal Intermittency in Granular Flow through Bottlenecks
}

\author{
D. Gella and I. Zuriguelఠ \\ Departamento de Física y Matemática Aplicada, Facultad de Ciencias, Universidad de Navarra, 31080 Pamplona, Spain \\ J. Ortín \\ Departament de Física de la Matéria Condensada, Facultat de Física, Universitat de Barcelona, O8028 Barcelona, Spain \\ and Universitat de Barcelona Institute of Complex Systems, 08028 Barcelona, Spain
}

(Received 6 May 2019; revised manuscript received 25 September 2019; published 22 November 2019)

\begin{abstract}
We experimentally analyze the intermittent nature of granular silo flow when the discharge is controlled by an extracting belt at the bottom. We discover the existence of four different scenarios. For low extraction rates, the system is characterized by an on-off intermittency. When the extraction rate is increased the structure functions of the grains velocity increments, calculated for different lag times, reveal the emergence of multifractal intermittency. Finally, for very high extraction rates that approach the purely gravitational discharge, we observe that the dynamics become dependent on the outlet size. For large orifices the behavior is monofractal, whereas for small ones, the fluctuations of the velocity increments deviate from Gaussianity even for very large time lags.
\end{abstract}

DOI: 10.1103/PhysRevLett.123.218004

Intermittency is a characteristic signature of three-dimensional fully developed turbulence, in which bursts of intense motion show up within quiescent fluid flow. In that framework, intermittency leads to increasingly important deviations of the velocity gradients from Gaussian statistics at increasingly smaller scales: energy transfer towards small scales is responsible for the nonzero skewness of the probability distribution functions, while bursts of energy dissipation produce fat-tailed distributions with large kurtosis [1]. These statistical features—arising from anomalous correlations of the velocity fluctuations-are well described in the framework of multifractality [2,3].

A multifractal is a scale-invariant system formed by a hierarchy of multiple fractal sets. It is characterized by its singularity spectrum, i.e., the collection of all its fractal dimensions defining how the system evolves under changes of scale. The connection between multifractality and intermittency appears to have a wide range of applicability. Thus, the multifractal formalism has been successfully applied to study intermittency in capillary-gravity wave turbulence [4,5], Lagrangian velocities in porous media flows [6,7], capillary bursts of two-phase flows in fractured media [8], and the morphology of fracture fronts $[9,10]$. Although the underlying physics in these problems is essentially different from the physics of turbulence, the fact that these assorted systems share analogous scaling properties provides useful information about their spatiotemporal dynamics.

In this work the multifractal formalism is used to study dense granular flows which may exhibit different regimes as continuous motion, stick-slip, avalanches, or intermittency $[11,12]$. Although intermittent granular flow has been already studied experimentally [11-16], theoretically [17], and numerically [18-20], to the best of our knowledge multifractal intermittency has been observed only in a simulated system subjected to homogeneous quasistatic shearing [20].

We focus on the silo discharge, a system where the intermittent flow properties have been explored in relation with clogging [21-23]. In purely gravitational discharge, Thomas and Durian [23] observed a gradual variation of the statistical properties of the grains motion, from nonGaussian to Gaussian as the orifice size increased, but without any abrupt change that could indicate the existence of a critical outlet size above which the system never gets clogged.

The experimental system used in this work is similar to the one described in [24] where the effect of a conveyor belt velocity on clogging was studied. Here, we focus on the flow dynamics considering also large outlet sizes where clogging never happens. In brief, the device consists of a quasi-two-dimensional silo of $61.2 \times 160 \mathrm{~cm}^{2}$ made of two transparent and parallel glass sheets separated by two $4 \mathrm{~mm}$ thick long aluminum pieces, which play the role of silo walls. In this way the granular material (stainless steel spheres of $d_{p}=4 \mathrm{~mm}$ diameter) arranges in a single layer. The silo bottom is formed by two wedge-shaped pieces whose separation defines the outlet size $D$. The outflow rate is controlled by a conveyor belt with an extraction velocity range between $v_{b}=0.1$ and $16 \mathrm{~cm} / \mathrm{s}$, placed below the orifice at a distance of $6.7 \pm 0.3 \mathrm{~mm}$ from the silo base. With this setup we are able to study the intermittent properties of the flow associated with the outlet size reduction [23], but also the ones induced by the belt. To do that, we 


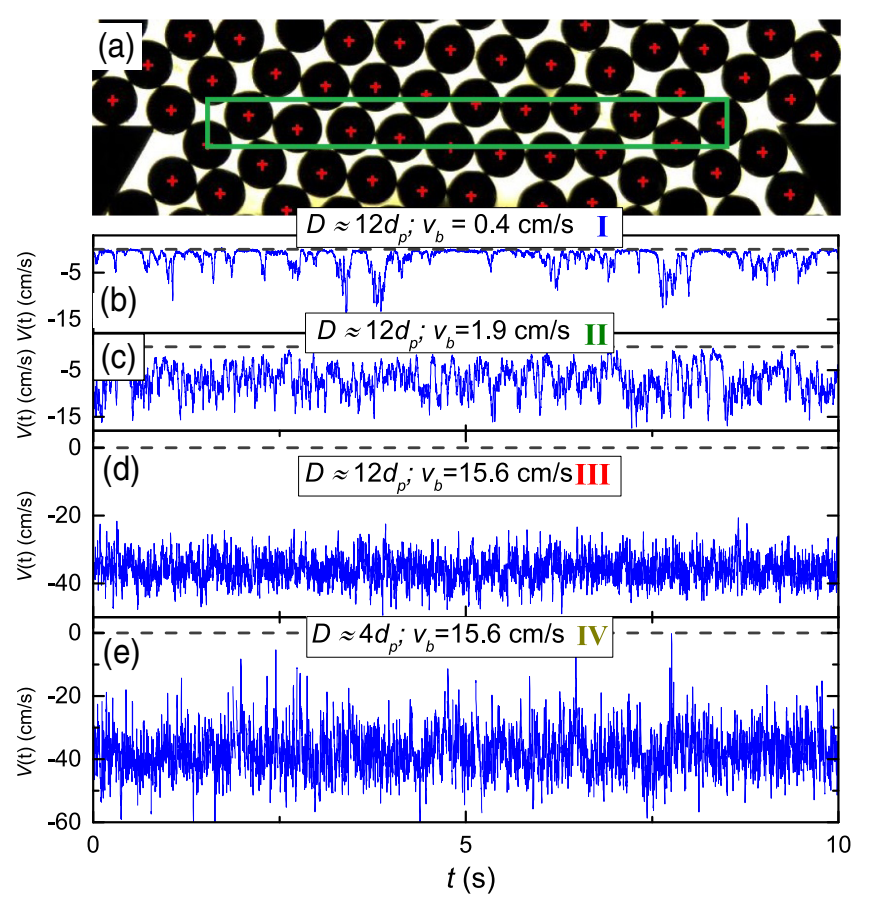

FIG. 1. (a) Frame extracted from one of the films recorded in the experiment. The red plus signs are the particles' centroids and the green rectangle indicates the region where the velocity vectors are averaged to obtain the velocity signals. (b)-(e) Velocity signals $V(t)$ for $D \approx 12 d_{p}$ and $D \approx 4 d_{p}$, and different values of $v_{b}$ as indicated in each panel. The dashed line indicates the zero level of $V(t)$.

explored all the $v_{b}$ ranges and two different outlet sizes: $D=1.61 \mathrm{~cm} \approx 4 d_{p}$ (where the formation of clogging is possible) and $D=4.81 \mathrm{~cm} \approx 12 d_{p}$ (where clogging never happens).

The flow of grains through the orifice was recorded discarding possible clog events with a frame rate of $800 \mathrm{fps}$ during $40 \mathrm{~s}$. We extracted images like the one in Fig. 1(a), and detected the particle centers and velocities in each frame. Then, a temporal velocity signal $V(t)$ is built by averaging the single vertical velocities corresponding to the beads positioned in a window centered at the orifice [green box in Fig. 1(a)]. Its vertical extent is one particle diameter whereas the horizontal one is $10 d_{p}$ for the case of $D \approx 12 d_{p}$ and $3 d_{p}$ for the case of $D \approx 4 d_{p}$. Figures $1(\mathrm{~b})-1(\mathrm{e})$ show examples of the $V(t)$ signals (32000 velocity data each). The distributions of the $V(t)$ values, whose uncertainty has been estimated of the order of $1 \mathrm{~cm} / \mathrm{s}$, are shown in the Supplemental Material [25].

The intermittency of $V(t)$ has been studied through a multifractal analysis [1] which requires the signals to be scale invariant. In order to prove this for our experimental $V(t)$, we tested their distributions of burst sizes $S$, defined as the area of the peaks under the signal mean value, following a procedure similar to that of Ref. [26]. Figure 2 displays the distributions of $S$ when they are normalized by

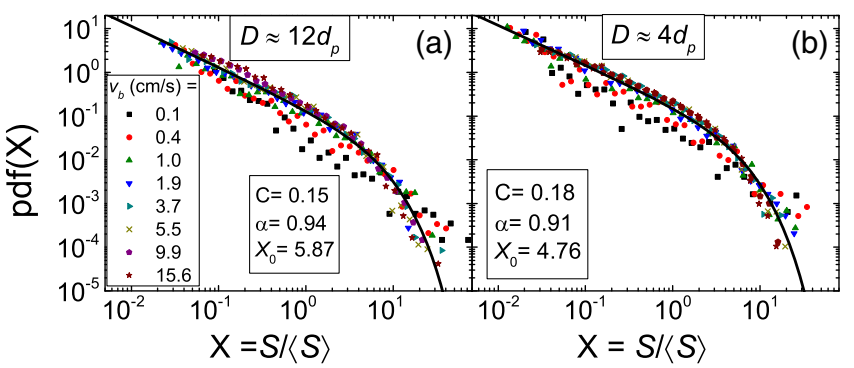

FIG. 2. Probability density functions of the normalized burst sizes $X=S /\langle S\rangle$ for $D \approx 12 d_{p}$ (a) and $D \approx 4 d_{p}$ (b), for the belt velocity values indicated in the legend. In both cases, the solid lines are the best fits of $P(X)=C X^{-\alpha} e^{-X / X_{0}}$ for the collapse of the data corresponding to $v_{b}=1.0 \mathrm{~cm} / \mathrm{s}$ and larger. The values of the fitting parameters used in each case appear inside the graphs.

the mean burst size, $X=S /\langle S\rangle$, showing a collapse of the curves for all but the two smallest values of $v_{b}$, for which only small deviations are observed. They are well described by the expression $P(X)=C X^{-\alpha} e^{-X / X_{0}}$, a power law with an exponential cutoff related to the system size, with similar fitting values of $C, \alpha$, and $X_{0}$ for both orifices, as shown inside the graphs. A power-law distribution of the burst sizes $S$ reflects that the signals $V(t)$ are scale invariant, and makes the multiscale analysis meaningful.

First, the structure functions of the velocity increments $\Delta V(\tau)=V(t+\tau)-V(t)$ are defined as $\mathcal{S}_{p}(\tau)=\left\langle|\Delta V(\tau)|^{p}\right\rangle$. In essence, this magnitude evaluates the $p$-order moments of the velocity increments $\Delta V(\tau)$ as functions of the lag time $\tau$. If the signal is scale invariant, the structure functions are proportional to power laws of the lag time $\mathcal{S}_{p}(\tau) \sim \tau^{\xi_{p}}$ [9]. The dependence of the exponent $\xi_{p}$ on $p$ determines the nature of the fluctuations: a linear trend $\xi_{p} \sim p$ indicates the presence of homogeneous time-independent fluctuations. Conversely, a nonlinear dependence implies the existence of inhomogeneous fluctuations leading to multifractal intermittency. In order to explore deviations from Gaussian statistics we computed the function $\mathcal{C}_{p}^{N}=\mathcal{C}_{p} / \mathcal{R}_{p}^{G}$ where $\mathcal{C}_{p}=\mathcal{S}_{p}^{1 / p}$ and $\mathcal{R}_{p}^{G}=\left(\mathcal{S}_{p}^{G}\right)^{1 / p} /\left(\mathcal{S}_{2}^{G}\right)^{1 / 2}$ is the ratio of structure functions for Gaussian distributions, a normalizing factor that depends only on $p$ [25]. In this way, Gaussian distributions would be reflected in similar values of $\mathcal{C}_{p}^{N}$ for all $p$.

Figures 3(a)-3(h) show log-log plots of the experimental structure functions for $D \approx 12 d_{p}$ (upper row) and $D \approx 4 d_{p}$ (lower row) and different values of $v_{b}$. Regarding the features of $\mathcal{C}_{p}^{N}$ in each case, we grouped the different behaviors obtained in four scenarios. The first one (I) is found for very low extraction rates and both outlet sizes [Figs. 3(a) and 3(e)]. The normalized structure functions exhibit increasing trends for low $\tau$, but they do not collapse in a single curve for high $\tau$, indicating that the distributions are not normal. We attribute this feature to the fact that 


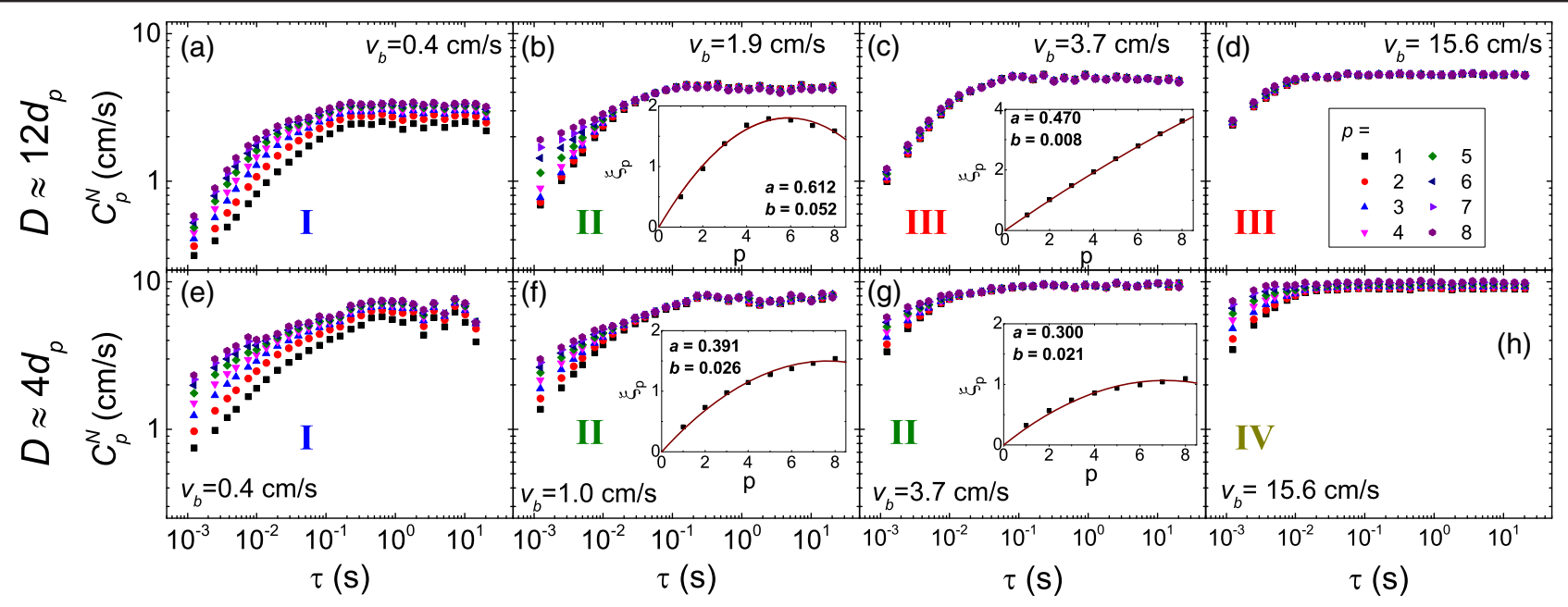

FIG. 3. (a)-(h) Sets of structure functions $C_{p}^{N}$ [ $p$ from 1 to 8 as indicated in the legend of (d)] for different outlet sizes and belt velocities. The roman numbers indicate the different scenarios as explained in the text. The insets in (b),(c),(f),(g) show the values of $\xi_{p}$ vs $p$ for the corresponding scenarios. The continuous lines in these insets correspond to second degree polynomial fits with $\xi_{p}=a p-b p^{2}$ and the parameters displayed in each plot.

$V(t)=0$ during long lapses of time, as evidenced in Fig. 1(b). Indeed, the $V(t)$ signal is displaying an on-off dynamics [1]: intervals of particle flow alternate with complete arrests. This behavior, which is intermittent per se, is compatible with the lack of collapse of the curves corresponding to the lowest values of $v_{b}$ reported in Fig. 2. The multifractal analysis is not allowed in these cases due to the lack of scale invariance, and so the structure functions do not follow a recognizable trend.

The second (II) scenario appears when the values of $v_{b}$ are increased [Figs. 3(b), 3(f), and 3(g)]. In these graphs, the $\mathcal{C}_{p}^{N}$ functions collapse for large $\tau$, revealing that all the moments become Gaussian for long time lags. Conversely, for short $\tau$ the different $p$-order $\mathcal{C}_{p}^{N}$ functions in power laws of the form $\mathcal{C}_{p}^{N} \sim \tau^{\xi_{p}} / p$ from which the value of $\xi_{p}$ for each $p$ has been obtained from power law fits. The dependence between these two parameters-shown in the insets of Figs. 3(b), 3(f), and 3(g) for their respective cases-has been successfully adjusted to second degree polynomial functions with the form $\xi_{p}=a p-b p^{2}$. As we pointed out before, this nonlinear trend implies the existence of multifractal intermittency. An example of a $V(t)$ signal for these conditions is displayed in Fig. 1(c).

The third (III) scenario is found for the big $\left(D \approx 12 d_{p}\right)$ outlet size and intermediate to large values of $v_{b}$ [Figs. 3(c) and 3(d)]. In this case all the $\mathcal{C}_{p}^{N}$ curves collapse in a single one, revealing the absence of multifractality since the $\xi_{p}$ dependence on $p$ becomes essentially linear [see inset of Fig. 3(c) and the very low value of the second order parameter]. These features are found for $V(t)$ signals like the one of Fig. 1(d).

Finally, the fourth (IV) scenario emerges for the small outlet size and large extraction rates [Fig. 3(h)]. Surprisingly, and in contrast with scenarios II and III, the normalized structure functions do not collapse even for the highest values of $\tau$. As the $V(t)$ dynamics [Fig. 1(e)] do not present the on-off intermittency observed in scenario I, we attribute this new behavior to non-Gaussian velocity fluctuations at all time lags.

In order to confirm the results obtained from the structure functions, we have calculated the normalized probability distributions of the velocity increments for different $\tau$ and different sets of parameters representing the four observed scenarios [Figs. 4(a)-4(f)]. In scenario I [Figs. 4(a)-4(d)] the distributions show a significant peak in 0. Remarkably, this singularity does not disappear for any value of $\tau$ confirming the aforementioned on-off intermittent behavior. Conversely, Figs. 4(b) and 4(e) (scenario II) show an evolution from non-Gaussian to Gaussian distributions as $\tau$ increases. This change of the statistical properties with $\tau$, which is a characteristic of multifractality, disappears in scenario III [Fig. 4(c)], where all the distributions practically follow the Gaussian function for all $\tau$ (see continuous line). A more complex behavior is gathered in Fig. 4(f) (scenario IV). Therein, the pdfs also evolve to a single one for long $\tau$, but in this case they do not completely reach a Gaussian behavior since the tails remain above the Gaussian distribution for all values of the lag time.

Aiming at a better characterization of the distribution deviations from Gaussianity, in Figs. 4(g)-4(i) we represent the kurtosis $K$ vs $\tau$ for the same experimental conditions than in Figs. 4(a)-4(f). In scenario I [Fig. 4(g)], the curves stay always above the Gaussian value, $K_{G}=3$. As $v_{b}$ is increased and scenario II is reached, the kurtosis exhibit decreasing trends at short $\tau$ and tend to $K_{G}$ for long $\tau$ [Fig 4(h)]. The value of $\tau$ at which $K$ reaches $K_{G}$ is a characteristic time $\tau_{c}$ above which the temporal fluctuations of $V(t)$ become Gaussian. Finally, Fig. 4(i) shows the kurtosis corresponding to scenarios III and IV. In the first 

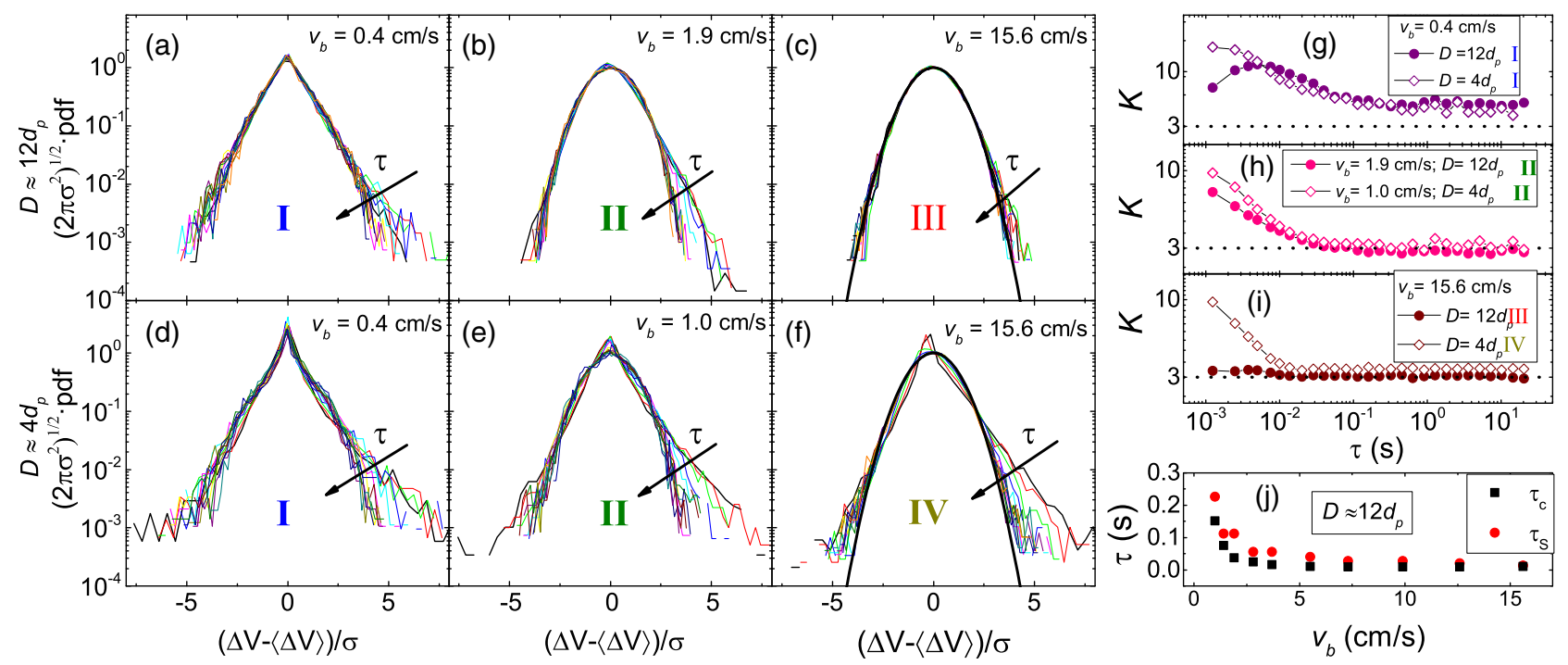

FIG. 4. (a)-(f) Sets of normalized probability density functions (pdf) of the velocity increments calculated with different $\tau$ for different values of $v_{b}$ and $D$. The solid lines in (c) and (f) are Gaussian plots. (g)-(i) Kurtosis of the previous pdfs as function of $\tau$. The dotted line corresponds to the Gaussian kurtosis $K_{G}=3$. (j) Characteristic lag times $\tau_{c}$ and $\tau_{S}$ as function of $v_{b}$ for $D \approx 12 d_{p}$, obtained as explained in the text. The roman numbers indicate the different scenarios according to the text.

case ( $D \approx 12 d_{p}$, full symbols) the deviation from the pure Gaussian statistics $\left(K=K_{G}\right)$ is very small and is only found for the smallest values of $\tau$. In the second $\left(D \approx 4 d_{p}\right.$, empty symbols), the values of $K$ at short $\tau$ follow a similar behavior than for scenario II, but for long $\tau$ the values keep a little above $K_{G}$ indicating that the distributions are leptokurtic. Although this deviation from $K_{G}$ is subtle, it is robust and reproducible, and implies the existence of an intermittent motion of a different nature as it will be explained further down.

From the graphs of Figs. 4(g)-4(i), the values of $\tau$ for which the distributions become Gaussian, $\tau_{c}$, are obtained by adjusting power laws for short $\tau$ values and calculating their intercepts with $K=K_{G}$. Furthermore, the time lag $\tau_{S}$ at which the structure functions of Fig. 3 become constant,

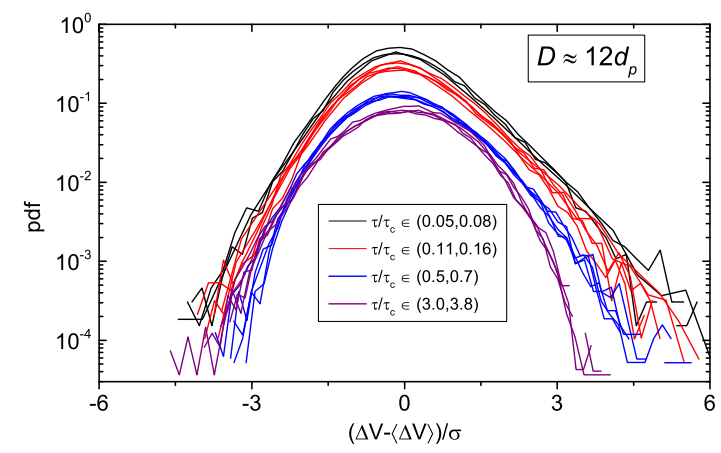

FIG. 5. Collapses of the normalized probability density functions for $D \approx 12 d_{p}$ and different values of $v_{b}$ and $\tau$. The curves appear grouped by colors according to the intervals of $\tau / \tau_{c}$ indicated in the legend. The distributions have been shifted in the vertical direction to allow better visualization. which reflects the loss of temporal correlation in the particles movement, have been also determined. The results of both characteristic times vs $v_{b}$ are shown in Fig. 4(j) for the case of $D \approx 12 d_{p}$ [27]. Both timescales $\tau_{c}$ and $\tau_{S}$ follow the same trend, increasing significantly as $v_{b}$ decreases, and seeming to diverge at a value of $v_{b}$ that corresponds to the frontier with region I. For high $v_{b}$, instead, both $\tau_{S}$ and $\tau_{c}$ tend to a constant value that should be related to the particle collisions time in the limit of large extraction rate. These situations (in which $\tau_{c}$ has saturated and does not depend on $v_{b}$ ) are the ones corresponding to scenario III. In these conditions the presence of the belt becomes unimportant and the regime resembles a traditional discharge (solely under gravity).

In Fig. 5 we plot all the distributions of velocity increments $\Delta V(\tau)$ for different $\tau$ and the values of $v_{b}$ corresponding to scenarios II and III. We observe a significant collapse of the pdfs with a similar value of $\tau / \tau_{c}$ (represented with the same color). This reveals that, for the cases included in scenarios II and III, we can characterize the intermittent features of the distributions with only one parameter, the ratio $\tau / \tau_{c}$. Also, Fig. 5 clearly shows the Gaussian shape of the distributions when $\tau / \tau_{c} \geq 3$, whereas at lower $\tau / \tau_{c}$ fat tails emerge as well as significant asymmetries (which are studied through the skewness [25]).

In summary, we prove that the flow in granular silo discharged by means of a conveyor belt exhibits different behavior depending on the experimental conditions. For low belt velocities the material is stopped during significant time intervals displaying an on-off intermittency (scenario I) with spatial correlations that span over the whole orifice [25]. As the extraction velocity increases, the system enters 
in a state characterized by the existence of multifractal intermittency (scenario II). Finally, for very high belt velocities we find two different scenarios. For large outlet sizes the fluctuations become homogeneous and Gaussian statistics are gathered regardless of the lag time considered (scenario III). For small outlet sizes, however, nonGaussian fluctuations appear even for the longest $\tau$ considered (scenario IV). This regime is attributed to fluctuations yielded by the formation of unstable grain structures above the outlet, which are only significant for small values of $D$. Importantly, the transition between scenarios III and IV is analogous to the one studied by Thomas and Durian in silos discharged solely by gravity [23] who argued that, indeed, this is not a proper transition in the sense that it does not occur for a given (critical) outlet size. Instead, the intermittent features emerge gradually as the outlet size decreases, a feature that was therefore connected with the nature of the clogging transition [28].

[1] U. Frisch, Turbulence: The Legacy of A.N. Kolmogorov (Cambridge University Press, Cambridge, England, 1995).

[2] R. Benzi, L. Biferale, G. Paladin, A. Vulpiani, and M. Vergassola, Phys. Rev. Lett. 67, 2299 (1991).

[3] K. R. Sreenivasan, Annu. Rev. Fluid Mech. 23, 539 (1991).

[4] E. Falcon, S. Fauve, and C. Laroche, Phys. Rev. Lett. 98, 154501 (2007).

[5] E. Falcon, S. G. Roux, and C. Laroche, Europhys. Lett. 90, 50007 (2010).

[6] P. de Anna, T. Le Borgne, M. Dentz, A. M. Tartakovsky, D. Bolster, and P. Davy, Phys. Rev. Lett. 110, 184502 (2013).

[7] V. L. Morales, M. Dentz, M. Willmann, and M. Holzner, Geophys. Res. Lett. 44, 9361 (2017).

[8] X. Clotet, J. Ortín, and S. Santucci, Phys. Rev. Lett. 113, 074501 (2014).

[9] N. Mallick, P.-P. Cortet, S. Santucci, S. G. Roux, and L. Vanel, Phys. Rev. Lett. 98, 255502 (2007).

[10] S. Santucci, K. J. Måløy, A. Delaplace, J. Mathiesen, A. Hansen, J. Ø. H. Bakke, J. Schmittbuhl, L. Vanel, and P. Ray, Phys. Rev. E 75, 016104 (2007).
[11] Y. Bertho, F. Giorgutti-Dauphine, and J. P. Hulin, Phys. Fluids 15, 3358 (2003).

[12] R. Fischer, P. Gondret, and M. Rabaud, Phys. Rev. Lett. 103, 128002 (2009).

[13] X-1. Wu, K. J. Måløy, A. Hansen, M. Ammi, and D. Bideau, Phys. Rev. Lett. 71, 1363 (1993).

[14] T. Le Pennec, K. J. Måløy, A. Hansen, M. Ammi, D. Bideau, and X. L. Wu, Phys. Rev. E 53, 2257 (1996).

[15] H. Kellay, Y. Amarouchene, and J. F. Boudet, Phys. Fluids 19, 078104 (2007).

[16] C. A. Álvarez and E. de Moraes Franklin, Physica (Amsterdam) 465A, 725 (2017).

[17] D. Helbing, A. Johansson, J. Mathiesen, M. H. Jensen, and A. Hansen, Phys. Rev. Lett. 97, 168001 (2006).

[18] S. Manna and H. Hermann, Eur. Phys. J. E 1, 341 (2000).

[19] P. Jalali, M. Li, J. Ritvanen, and P. Sarkoma, Chaos 13, 434 (2003).

[20] F. Radjai and S. Roux, Phys. Rev. Lett. 89, 064302 (2002).

[21] E. Longhi, N. Easwar, and N. Menon, Phys. Rev. Lett. 89, 045501 (2002).

[22] A. Janda, R. Harich, I. Zuriguel, D. Maza, P. Cixous, and A. Garcimartín, Phys. Rev. E 79, 031302 (2009).

[23] C. C. Thomas and D. J. Durian, Phys. Rev. E 94, 022901 (2016).

[24] D. Gella, I. Zuriguel, and D. Maza, Phys. Rev. Lett. 121, 138001 (2018).

[25] See Supplemental Material at http://link.aps.org/ supplemental/10.1103/PhysRevLett.123.218004 for the pdfs of the velocity signals, the skewness of the velocity increments distributions, a detailed explanation of the structure functions normalization, and the correlation length in the particles' motion.

[26] R. Planet, S. Santucci, and J. Ortín, Phys. Rev. Lett. 102, 094502 (2009).

[27] For the case of $D \approx 4 d_{p}$ this analysis has not been carried out since the evolution to Gaussian distributions for large $\tau$ is only reached in a very small range of $v_{b}$.

[28] C. C. Thomas and D. J. Durian, Phys. Rev. Lett. 114, 178001 (2015). 